\title{
Incidence and prognosis of myocardial injury in patients with severe trauma
}

\author{
Alexandra Stroda ${ }^{1} \cdot$ Simon Thelen ${ }^{2}(1) \cdot$ René M'Pembele $^{1} \cdot$ Antony Adelowo $^{1} \cdot$ Carina Jaekel $^{2} \cdot$ Erik Schiffner $^{2}$. \\ Dan Bieler ${ }^{2} \cdot$ Michael Bernhard $^{3} \cdot$ Ragnar Huhn $^{1} \cdot$ Giovanna Lurati Buse $^{1} \cdot$ Sebastian Roth ${ }^{1}$
}

Received: 28 September 2021 / Accepted: 22 November 2021 / Published online: 8 December 2021

(c) The Author(s) 2021

\begin{abstract}
Purpose Severe trauma can lead to end organ damages of varying severity, including myocardial injury. In the non-cardiac surgery setting, there is extensive evidence that perioperative myocardial injury is associated with increased morbidity and mortality. The impact of myocardial injury on outcome after severe trauma has not been investigated adequately yet. We hypothesized that myocardial injury is associated with increased in-hospital mortality in patients with severe trauma.

Materials/methods This retrospective cohort study included patients $\geq 18$ years with severe trauma [defined as injury severity score (ISS) $\geq 16$ ] that were admitted to the resuscitation room of the Emergency Department of the University Hospital Duesseldorf, Germany, between 2016 and 2019. The main endpoint was in-hospital mortality. Main exposure was myocardial injury at arrival [defined as high-sensitive troponin $\mathrm{T}(\mathrm{hsTnT})>14 \mathrm{ng} / \mathrm{l}$ ]. For statistical analysis, receiver operating characteristic curve (ROC) and multivariate binary logistic regression were performed.

Results Out of 368 patients, 353 were included into statistical analysis ( $72.5 \%$ male, age: $55 \pm 21$, ISS: $28 \pm 12$ ). Overall inhospital mortality was 26.1\%. Myocardial injury at presentation was detected in $149(42.2 \%)$ patients. In-hospital mortality of patients with and without myocardial injury at presentation was $45 \%$ versus $12.3 \%$, respectively. The area under the curve (AUC) for hsTnT and mortality was 0.76 [95\% confidence interval (CI) $0.71-0.82$ ]. The adjusted odds ratio of myocardial injury for in-hospital mortality was 2.27 ([95\%CI 1.16-4.45]; $p=0.017$ ).

Conclusion Myocardial injury after severe trauma is common and independently associated with in-hospital mortality. Thus, hsTnT might serve as a new prognostic marker in this cohort.
\end{abstract}

Keywords Troponin $\cdot$ Cardiac biomarkers $\cdot$ Resuscitation room $\cdot$ Multiple trauma $\cdot$ Mortality

Simon Thelen

Simon.Thelen@med.uni-duesseldorf.de

1 Department of Anesthesiology, Medical Faculty and University Hospital Duesseldorf, Heinrich-HeineUniversity Duesseldorf, Moorenstr. 5, 40225 Duesseldorf, Germany

2 Department of Orthopedics and Trauma Surgery, Medical Faculty and University Hospital Duesseldorf, Heinrich-Heine-University Duesseldorf, Moorenstr. 5, 40225 Duesseldorf, Germany

3 Emergency Department, Medical Faculty and University Hospital Duesseldorf, Heinrich-Heine-University Duesseldorf, Moorenstr. 5, 40225 Duesseldorf, Germany

\section{Introduction}

Severe trauma accounts for a great number of deaths worldwide and still is one of the leading causes of death among those under 40 years [1]. As the incidence of severe trauma is high, prediction of outcome is still a subject of current research. In this context, several prognostic scores, such as the revised trauma score (RTS) or the trauma and injury severity score (TRISS) have been suggested [2-5]. However, these scores do not take into account end organ damages apart from trauma-associated physical injuries which might also play a substantial role. One of these end organ damages in severely injured patients might be myocardial injury. According to the fourth universal definition of myocardial infarction, any detection of troponin exceeding the 99th percentile represents myocardial injury, independent of its pathophysiological mechanism [6]. In the non-cardiac 
surgery setting, there is extensive evidence that perioperative myocardial injury is associated with higher mortality and an increased rate of postoperative major adverse cardiovascular events (MACE) [7]. In addition to direct chest trauma, several potential other pathomechanisms postulated myocardial injury after surgery, i.e., hemodynamic disturbances due to acute bleeding, as well as activation of coagulation and inflammation. These mechanisms are also present in trauma [8-10]. As high-sensitivity troponin assays are able to detect troponin release less than $1 \mathrm{~h}$ after an initial event, measurement of troponin at presentation after trauma might detect both, direct myocardial injury by immediate chest trauma and secondary myocardial injury due to a mismatch of oxygen supply and demand. The impact of myocardial injury on outcome after severe trauma is underexplored. Therefore, we hypothesized that myocardial injury at hospital presentation regardless of its cause is associated with increased in-hospital mortality after severe trauma.

\section{Methods}

The present study is a retrospective, single-center cohort study, approved by the ethical committee of the HeinrichHeine-University Duesseldorf, Germany (Reference number 2020-1122). The study was conducted in accordance with the guidelines for good clinical practice (GCP) and the declaration of Helsinki. All handling of personal data complied with the GCP Guidelines and followed the General Data Protection Regulation (EU) 2016/679. This manuscript follows the STROBE reporting guidelines for retrospective cohort studies. The research question was formulated in compliance with the PICO format.

\section{Participants}

Severely injured patients [defined as injury severity score (ISS) $\geq 16$ ] and patients who were $\geq 18$ years of age admitted to the resuscitation room of the Emergency Department of the University Hospital Duesseldorf (Level I trauma center) between January 2016 and December 2019 were included in the study $[11,12]$. Patients were excluded when no measurement of troponin at arrival in the resuscitation room was available. The treatment of all patients was standardized according to Advanced Trauma Life Support (ATLS) guidelines.

\section{Exposures and main endpoint}

The main exposure was myocardial injury as defined by the fourth universal definition of myocardial infarction, detected using high-sensitive troponin T (hsTnT; Roche Diagnostics, Elecsys $\left.{ }^{\circledR}\right)[6]$. HsTnT was measured immediately after arrival in the resuscitation room (main exposure) as well as on day $1(=24 \mathrm{~h}$ after trauma) and day $2(=48 \mathrm{~h}$ after trauma) (secondary exposures). The main endpoint of this study was in-hospital mortality.

\section{Sample size}

Due to the retrospective nature of the study, we did not conduct a formal sample size calculation. Between 2016 and 2019 , on average approximately 100 patients/year met the inclusion criteria and were defined as having adult severe trauma (ISS $\geq 16$ ). Based on the current literature, we expected an all-cause in-hospital mortality of approximately $25 \%$ ( $=100$ expected events in total) [13]. Assuming that hsTnT values would be available in $>90 \%$ of patients, a sample size of 360 patients was deemed appropriate to answer the research question including robust multivariable adjustment with nine co-variables according to Peduzzi et al. [14].

\section{Statistical analysis}

A complete case analysis was conducted. Categorical data were presented as absolute numbers (percent). Continuous data were presented as mean \pm standard deviation or median (interquartile range) as applicable. The discrimination of hsTnT for in-hospital mortality was examined by receiver operating characteristic curve (ROC) and the area under the curve (AUC). To quantify the association between myocardial injury and the main endpoint, multivariate binary logistic regression with forced entry of predefined co-variables was conducted. For continuous variables, the established cutoff (hsTnT) or data-driven cutoff (age, base excess) was used. The coding and definitions for each covariable can be found in supplementary Table S3. Hosmer-Lemeshow test was performed to evaluate goodness of fit. In addition, we evaluated the additional value of myocardial injury when added to the ISS score in a separate logistic regression model. We also calculated the AUCs for all logistic regression models with and without myocardial injury to determine potential improvement and performed De Long-Test for comparison of AUCs. During review process, a "postfactum" Kaplan-Meier analysis was performed.

\section{Predefined co-variables}

The following co-variables for multivariable adjustment were predefined: age, sex, ISS, pre-existing chronic kidney injury [as defined by Kidney Disease Improving Global Outcome (KDIGO) Criteria], pre-existing coronary artery disease, base excess[15], American Society of Anesthesiologists (ASA) classification and thorax trauma (defined as any form of physical injury to the chest including the ribs, muscles, heart and lungs). The choice of co-variables was 
driven by its potential impact on both troponin and outcome and based on literature research by two independent members of the study team.

\section{Results}

In total, 368 patients were screened for inclusion, thereof 15 patients had to be excluded due to missing hsTnT values at admission to the resuscitation room of the Emergency Department. Data on the main endpoint and all other covariables were complete (see Fig. 1). Patient characteristics are presented in Table 1. Mean hsTnT at presentation was $63.33 \pm 415.72$. At arrival, 149/353 (42.2\%) patients presented with myocardial injury (hsTnT $>14 \mathrm{ng} / \mathrm{l})$. Overall in-hospital mortality was $26.1 \%$ (92 / 353 patients). Mortality was $45 \%$ (67 of 149 patients) versus $12.3 \%$ ( 25 of 204 patients) among patients with and without myocardial injury at presentation, respectively. 179/353 (50.7\%) patients had thorax trauma. In patients with myocardial injury, thorax trauma was present in $81 / 149$ (54.4\%) patients. Due to missing values or death, 108/353 (30.6\%) patients had hsTnT measurement on day 1 and 110/353 (31.2\%) patients on day 2.

\section{ROC analysis}

The AUC of initial hsTnT for in-hospital mortality was 0.76 [95\% confidence interval (CI) 0.71-0.82] (see Fig. 2). The AUC for hsTnT and in-hospital mortality on day 1 and 2 was 0.84 [95\% CI 0.75-0.92] and 0.87 [95\% CI 0.79-0.95], respectively (see Fig. 3).

\section{Multivariate binary logistic regression}

Multivariate binary logistic regression revealed an adjusted odds ratio (OR) of 2.27 [95\% CI 1.16-4.45] for myocardial injury and in-hospital mortality (see Table 2). Hosmer-Lemeshow test revealed a $p$ value of 0.3 . The full results of multivariate analysis are reported in Table 2 . The results of univariate analysis for each covariable are presented in supplementary Table S1.

\section{AUC of logistic regression models}

The AUC for the above described logistic regression model was 0.85 [95\% CI $0.80-0.90]$ without myocardial injury and 0.86 [95\% CI 0.81-0.90] including myocardial injury. De Long test showed that the difference between the AUCs was not significant.

\section{Additional value of myocardial injury when added to ISS Score}

The AUC of a univariate logistic regression model including ISS and in-hospital mortality was 0.67 [95\% CI 0.60-0.73]. The AUC improved to 0.76 [95\% CI 0.71-0.82] when adding myocardial injury to this model. According to De Long Test, this improvement was significant with a difference between AUCs of 0.09 ([95\% CI $0.04-0.15]$; $p=0.0007$ ).

\section{Survival analysis}

Kaplan-Meier analysis revealed that in-hospital survival rates of patients with and without myocardial injury are significantly different $(p<0.001$, see supplementary Figure S1).
Fig. 1 Study flowchart showing selection process of the study cohort

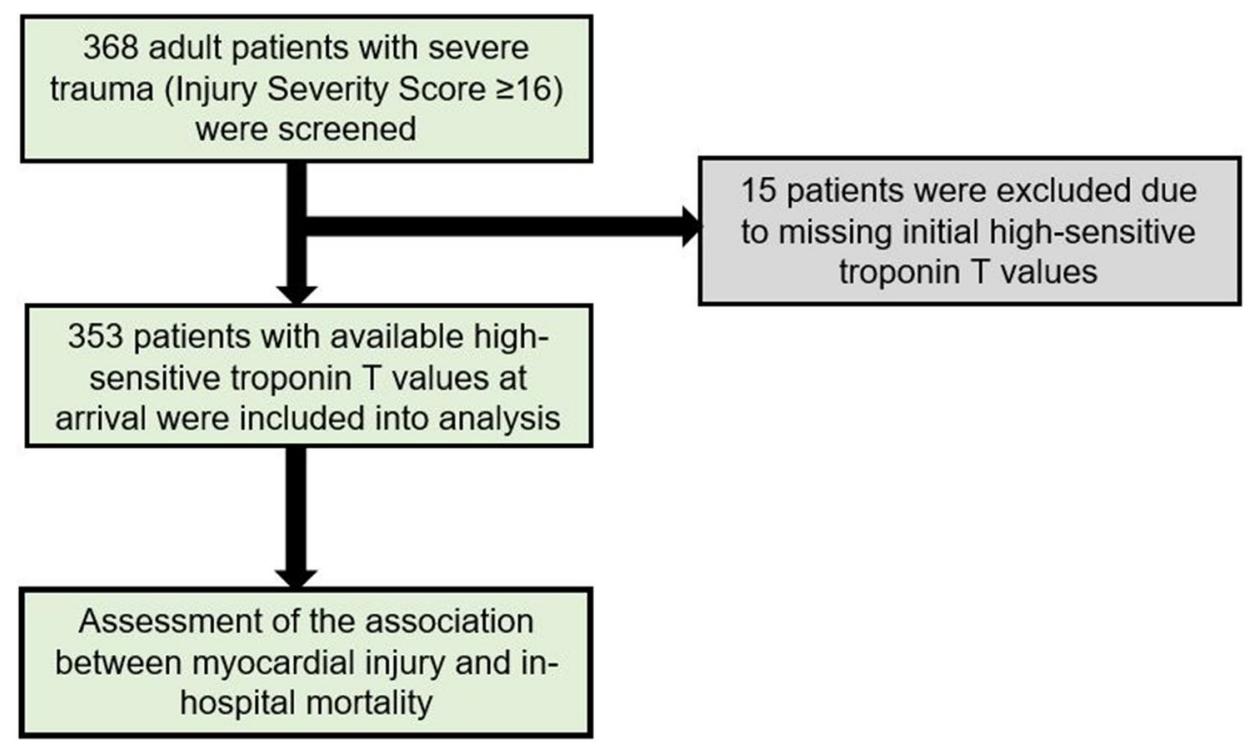


Table 1 Patient characteristics $\begin{array}{ll}\begin{array}{l}\text { Patients with severe trauma } \\ (n=353)\end{array} & \begin{array}{l}\text { Patients with initial myocardial } \\ \text { injury }(n=149)\end{array}\end{array}$
Patients without initial myocardial injury $(n=204)$

\begin{tabular}{|c|c|c|c|}
\hline \multicolumn{4}{|l|}{ Baseline characteristics } \\
\hline Male sex no. $(\%)$ & $256(72.5 \%)$ & $104(69.8 \%)$ & $152(74.5 \%)$ \\
\hline Age (years) & $55 \pm 21$ & $62 \pm 22$ & $50 \pm 18$ \\
\hline \multicolumn{4}{|l|}{ Comorbidities } \\
\hline Coronary artery disease & $28(7.9 \%)$ & $16(10.7 \%)$ & $12(5.9 \%)$ \\
\hline Chronic kidney disease ( $\geq$ CKD III) & $9(2.5 \%)$ & $7(4.7 \%)$ & $2(1.0 \%)$ \\
\hline Diabetes mellitus & $22(6.2 \%)$ & $9(6.0 \%)$ & $13(6.4 \%)$ \\
\hline Arterial hypertension & $85(24.1 \%)$ & $41(27.5 \%)$ & $44(21.6 \%)$ \\
\hline \multicolumn{4}{|l|}{ ASA physical status } \\
\hline$A S A I$ & $159(45 \%)$ & $47(31.5 \%)$ & $112(54.9 \%)$ \\
\hline ASA II & $111(31.4 \%)$ & $57(38.3 \%)$ & $54(26.5 \%)$ \\
\hline ASA III & $52(14.7 \%)$ & $24(16.1 \%)$ & $28(13.7 \%)$ \\
\hline ASA IV & $7(2.0 \%)$ & $5(3.4 \%)$ & $2(1.0 \%)$ \\
\hline \multicolumn{4}{|l|}{ Trauma related data } \\
\hline ISS & $28 \pm 12$ & $31 \pm 12$ & $26 \pm 10$ \\
\hline GCS at arrival & $3[3-14]$ & $3[3-9]$ & $10[3-15]$ \\
\hline Thorax trauma & $179(50.7 \%)$ & $81(54.4 \%)$ & $98(48.0 \%)$ \\
\hline \multicolumn{4}{|l|}{ Laboratory values } \\
\hline $\mathrm{Hb}(\mathrm{mg} / \mathrm{dl})$ & $12.3 \pm 2.4$ & $11.6 \pm 2.5$ & $12.8 \pm 2.1$ \\
\hline INR & $1.4 \pm 0.8$ & $1.6 \pm 1.0$ & $1.2 \pm 0.5$ \\
\hline PTT (sec) & $31.75 \pm 24.4$ & $38.0 \pm 32.6$ & $27.3 \pm 15.0$ \\
\hline Base excess & $-3.8 \pm 5.7$ & $-5.6 \pm 6.7$ & $-2.5 \pm 4.5$ \\
\hline HsTnT initial (ng/ml) & $63.33 \pm 415.72$ & $139.8 \pm 633.1$ & $7.46 \pm 3.0$ \\
\hline Creatinine initial $(\mathrm{mg} / \mathrm{dl})$ & $1.04 \pm 0.62$ & $1.16 \pm 0.51$ & $0.95 \pm 0.68$ \\
\hline HsTnT 24 h (ng/ml) & $134.80 \pm 271.28$ & $199.40 \pm 332.06$ & $44.36 \pm 96.16$ \\
\hline HsTnt 48 h (ng/ml) & $477.75 \pm 2900.88$ & $865.75 \pm 4004.0$ & $60.45 \pm 177.85$ \\
\hline \multicolumn{4}{|l|}{ Outcome } \\
\hline Death in hospital & $92(26.1 \%)$ & $67(45 \%)$ & $25(12.3 \%)$ \\
\hline
\end{tabular}

Values are presented as $N(\%)$ or mean $( \pm \mathrm{SD})$ /median $(\mathrm{IQL})$, where appropriate ASA American Society of Anesthesiologists, ISS injury severity score, $g C S$ Glasgow Coma Scale, $H b$ hemoglobin, INR international normalized ratio, PTT partial thromboplastin time, HsTnT high-sensitive troponin

\section{Discussion}

The results of this study demonstrate that myocardial injury at arrival in the resuscitation room is common and independently associated with in-hospital mortality among severely injured patients. Furthermore, the addition of myocardial injury to the established ISS score was able to improve prognostic value significantly.

\section{Troponin as a predictor of mortality in severe trauma patients in the literature}

Varying pathomechanisms leading to myocardial injury in severe trauma patients are discussed $[9,10,16]$. To date, only few studies investigated this aspect in the past [17-19]. Keskpaik et al. retrospectively investigated 147 severely injured patients with chest trauma [defined as Chest Abbreviated Injury Scale (AIS) > 3][17]. The primary endpoints of this study were in-hospital mortality and 1-year mortality. The authors found that elevated troponin is associated with poor outcome compared to patients with normal troponin levels. As only patients with chest trauma were included, a selection bias might be present in this study not considering alternative pathomechanisms such as a mismatch between oxygen supply and demand. Edouard et al. postulated in another analysis including 728 patients that elevated troponin I is not associated with higher mortality in trauma patients [20]. However, the authors concluded that troponin I assessment may be used for initial screening in high-risk trauma patients to detect anatomical cardiac injuries. While interpreting the findings listed above, it has to be taken into account that definition of trauma and measurement of 


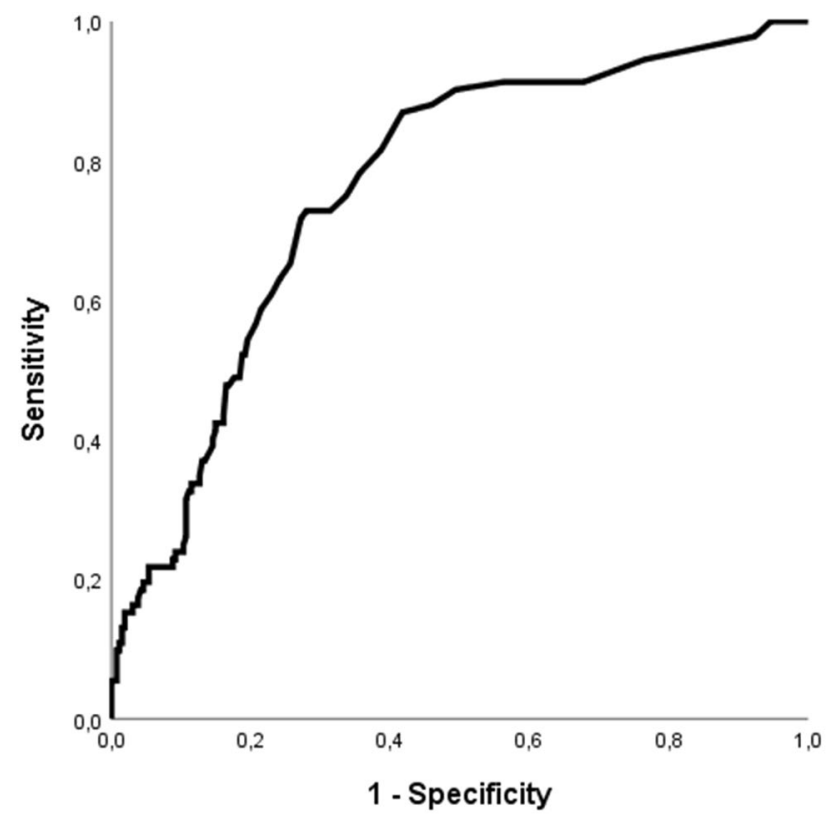

Fig. 2 Receiver operating characteristics (ROC) curve showing the discrimination of initial high-sensitive troponin $\mathrm{T}$ (hsTnT) for in-hospital mortality. ROC analysis revealed an AUC of 0.76 [95\% confidence interval (CI) 0.71-0.82]

\section{hsTnT on day 1}

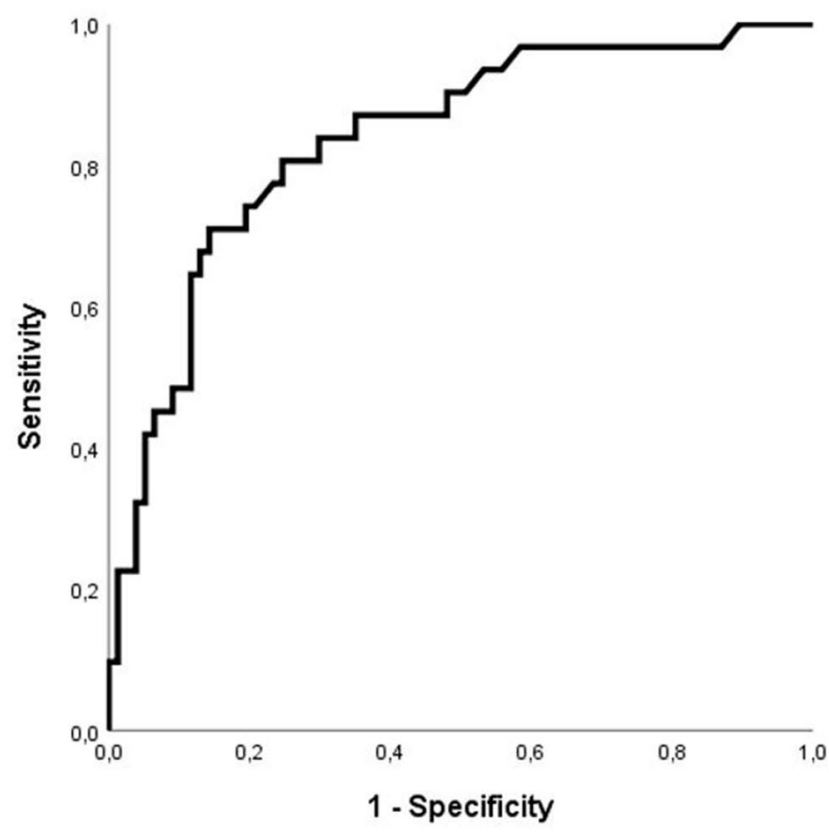

Fig. 3 Receiver operating characteristics (ROC) curves showing the discrimination of high-sensitive troponin $\mathrm{T}(\mathrm{hsTnT})$ on day 1 and 2 for in-hospital mortality. ROC analysis of hsTnT on day 1 revealed an troponin (troponin I versus troponin T) differ between the examined cohorts. Moreover, Edouard et al. did not give clear definitions of "late mortality" and trauma severity and did only distinguish between troponin release yes/no rather than considering troponin as continuous variable. Mahmood et al. also identified troponin as a risk marker for mortality in severe trauma patients, but did predominantly concentrate on patients with chest trauma, in whom traumatic damage of the heart plays a crucial role [17, 21]. The authors performed a retrospective analysis of 993 blunt traumatic chest injury patients divided into two groups (positive vs. negative troponin $\mathrm{T}$ ) and concluded that patients with elevated troponin tend to have the worst outcome even in the absence of clinical evidence of acute cardiac involvement.

The findings of our study add to the limited literature in this field. We investigated a broad and representative cohort of patients with severe trauma. Especially, we included all types of injuries taking into account other mechanisms of troponin release than chest trauma, and according to our multivariate analysis, the association between myocardial injury and mortality was independent of chest trauma. Although we performed a retrospective analysis, our data are based on a prospectively constituted database which ensures high data quality. In addition, we did not only investigate initial troponin, but also included measurements on day 1 and 2 after trauma which enables the interpretation of troponin values over time. Finally, we evaluated the additional value

\section{hsTnT on day 2}

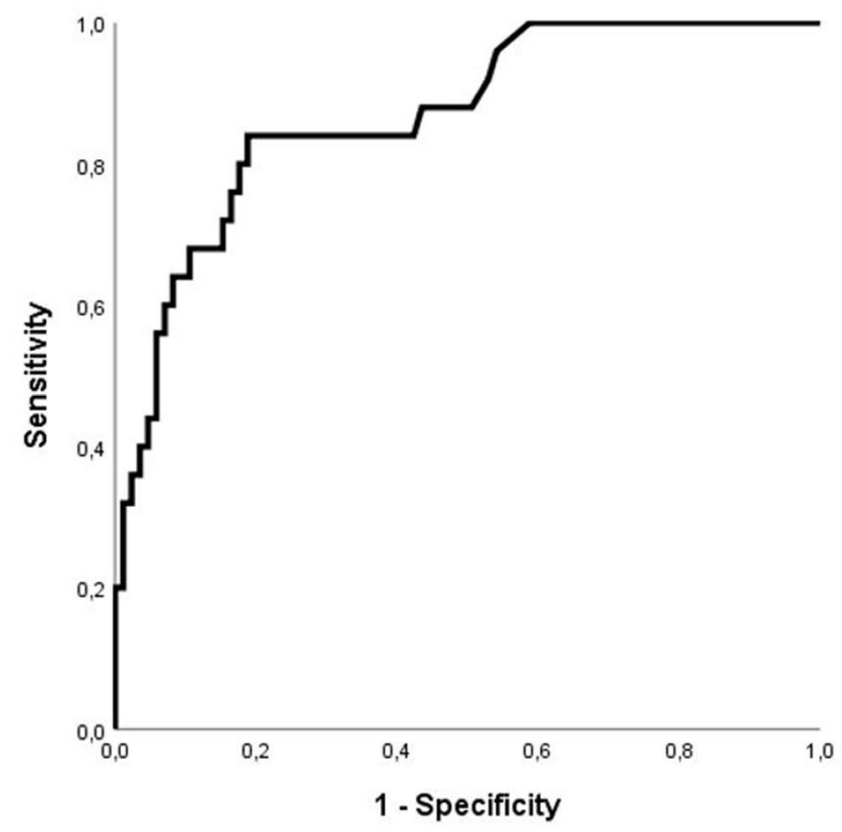

AUC of 0.84 [95\% CI 0.75-0.92]. The AUC of hsTnT on day 2 was 0.87 [95\% CI 0.79-0.95] 
Table 2 Multivariate binary logistic regression model

\begin{tabular}{lcllrr}
\hline Variable & $\begin{array}{l}\text { Regression coef- } \\
\text { ficient }\end{array}$ & Odds ratio & \multicolumn{2}{c}{$95 \%$ Confidence interval } & $p$ value \\
\cline { 4 - 5 } & & & Lower & Upper & \\
\hline Age & 1.611 & 5.01 & 2.35 & 10.70 & $<\mathbf{0 . 0 0 1}$ \\
Myocardial Injury & 0.82 & 2.27 & 1.16 & 4.45 & $\mathbf{0 . 0 1 7}$ \\
ISS Score & 0.052 & 1.05 & 1.02 & 1.08 & $<\mathbf{0 . 0 0 1}$ \\
Sex & -0.07 & 0.93 & 0.45 & 1.90 & 0.833 \\
ASA physical status & -0.10 & 0.91 & 0.58 & 1.41 & 0.671 \\
Thorax trauma & -0.69 & 0.50 & 0.25 & 1.00 & 0.052 \\
Chronic kidney disease & -0.96 & 0.38 & 0.06 & 2.39 & 0.304 \\
Coronoary artery disease & 0.84 & 2.35 & 0.80 & 6.66 & 0.123 \\
Base excess & 1.78 & 5.63 & 2.83 & 11.20 & $<\mathbf{0 . 0 0 1}$ \\
Constant term & -4.3 & 0.01 & & & $<\mathbf{0 . 0 0 1}$ \\
\hline
\end{tabular}

Significant results are presented as bold $p$-values

ISS injury severity score, ASA American Society of Anesthesiologists of myocardial injury when added to the established ISS and our results show clearly that the combination of myocardial injury and ISS is significantly better in terms of prognosis than ISS alone.

\section{Strengths and limitations}

Our study has several strengths and limitations. Strengths: Because of the standardized collection of data in patients enrolled in the TraumaRegistry®, data quality of this study can be regarded as high. Moreover, we conducted robust multivariate regression including nine relevant covariates and the choice of these covariates was based on literature research. Limitations: One limitation of this study consists of the retrospective single-center design. However, our results for the main endpoint in-hospital mortality are in line with the current literature and thus can be considered as representative for our study cohort. A second limitation refers to ROC analysis for troponin and mortality on day 1 and 2 . It is important to clarify that mainly patients with elevated initial troponin at the resuscitation room might have received further troponin sampling which surely led to relevant selection bias. Third, this study only investigated the association between myocardial injury and in-hospital mortality. Future studies should investigate the association with further relevant endpoints (e.g. in-hospital complications, hospital length of stay or long-term outcome).

\section{Conclusions}

Myocardial injury after severe trauma is common and independently associated with in-hospital mortality. Thus, hsTnT may serve as a new prognostic marker in this cohort. These results are clinically relevant and troponin may be considered to be included into established risk scores for trauma patients.

Supplementary Information The online version contains supplementary material available at https://doi.org/10.1007/s00068-021-01846-2.

Acknowledgements None.

Author contributions AS: Investigation, data curation, formal analysis, writing-original draft; ST: conceptualization, resources, supervision, writing - review and editing; RMP: formal analysis, writing—review and editing; AA: investigation, data curation; CJ: conceptualization, resources, writing-review and editing; ES: conceptualization, resources, writing-review and editing; DB: conceptualization, resources, writing - review and editing; MB: conceptualization, writing-review \& editing; RH: conceptualization, supervision, writing-review and editing; GLB: conceptualization, methodology, formal analysis, supervision, writing-review and editing; SR: project administration, conceptualization, methodology, investigation, formal analysis, writing —original draft.

Funding Open Access funding enabled and organized by Projekt DEAL. This study received no external funding.

Availability of data and materials The datasets generated during and/ or analyzed during the current study are available from the first author on reasonable request.

\section{Declarations}

Conflicts of interest All authors declare that there are no conflicts of interest.

Ethics approval The study was approved by the ethical committee of the Heinrich-Heine-University, Duesseldorf, Germany (reference number 2020-1122).

Consent for publication/Consent to participate Not applicable.

Open Access This article is licensed under a Creative Commons Attribution 4.0 International License, which permits use, sharing, 
adaptation, distribution and reproduction in any medium or format, as long as you give appropriate credit to the original author(s) and the source, provide a link to the Creative Commons licence, and indicate if changes were made. The images or other third party material in this article are included in the article's Creative Commons licence, unless indicated otherwise in a credit line to the material. If material is not included in the article's Creative Commons licence and your intended use is not permitted by statutory regulation or exceeds the permitted use, you will need to obtain permission directly from the copyright holder. To view a copy of this licence, visit http://creativecommons. org/licenses/by/4.0/.

\section{References}

1. Destatis SB. Gestorbene: Deutschland, Jahre, Todesursachen, Altersgruppen. Accessed 25.08.2021, https://www.genesis.desta tis.de/genesis/onlineoperation $=$ previous \&levelindex $=2 \&$ step $=2 \&$ titel=Ergebnis\&levelid=1629917943886\&acceptscookies=false $\#$ abreadcrumb

2. Ahmad HN. Evaluation of revised trauma score in polytraumatized patients. J Coll Physicians Surg Pak. 2004;14(5):286-9.

3. Boyd CR, Tolson MA, Copes WS. Evaluating trauma care: the TRISS method. Trauma score and the injury severity score. J Trauma. 1987;27(4):370-8.

4. Lefering R, Huber-Wagner S, Nienaber U, Maegele M, Bouillon B. Update of the trauma risk adjustment model of the TraumaRegister DGU: the Revised Injury Severity Classification, version II. Crit Care. 2014;18(5):476. https://doi.org/10.1186/ s13054-014-0476-2.

5. Lefering R. Development and validation of the revised injury severity classification score for severely injured patients. Eur J Trauma Emerg Surg. 2009;35(5):437-47. https://doi.org/10.1007/ s00068-009-9122-0.

6. Thygesen K, Alpert JS, Jaffe AS, et al. Fourth universal definition of myocardial infarction. Kardiol Pol. 2018;76(10):1383-415. https://doi.org/10.5603/KP.2018.0203.

7. Puelacher C, Lurati Buse G, Seeberger D, et al. Perioperative myocardial injury after noncardiac surgery: incidence, mortality, and characterization. Circulation. 2018;137(12):1221-32. https://doi. org/10.1161/CIRCULATIONAHA.117.030114.

8. Weber B, Lackner I, Gebhard F, Miclau T, Kalbitz M. Trauma, a matter of the heart-molecular mechanism of post-traumatic cardiac dysfunction. Int J Mol Sci. 2021. https://doi.org/10.3390/ ijms22020737.

9. Martin M, Mullenix P, Rhee P, Belzberg H, Demetriades D, Salim A. Troponin increases in the critically injured patient: mechanical trauma or physiologic stress? J Trauma. 2005;59(5):1086-91. https://doi.org/10.1097/01.ta.0000190249.19668.37.

10. Wu TT, Yuan A, Chen CY, et al. Cardiac troponin I levels are a risk factor for mortality and multiple organ failure in noncardiac critically ill patients and have an additive effect to the APACHE II score in outcome prediction. Shock. 2004;22(2):95-101. https:// doi.org/10.1097/01.shk.0000132484.97424.32.

11. Copes WS, Champion HR, Sacco WJ, Lawnick MM, Keast SL, Bain LW. The injury severity score revisited. J Trauma. 1988;28(1):69-77. https://doi.org/10.1097/00005373-19880 1000-00010.

12. Blätzinger M. TraumaRegister DGU®. Accessed 20.09.2021, https://www.traumaregister-dgu.de

13. Ruchholtz S, Lefering R, Paffrath T, et al. Reduction in mortality of severely injured patients in Germany. Dtsch Arztebl Int. 2008;105(13):225-31. https://doi.org/10.3238/arztebl.2008.0225.

14. Peduzzi P, Concato J, Feinstein AR, Holford TR. Importance of events per independent variable in proportional hazards regression analysis II Accuracy and precision of regression estimates. J Clin Epidemiol. 1995;48(12):1503-10. https://doi.org/10.1016/ 0895-4356(95)00048-8.

15. Ibrahim I, Chor WP, Chue KM, et al. Is arterial base deficit still a useful prognostic marker in trauma? A systematic review. Am J Emerg Med. 2016;34(3):626-35. https://doi.org/10.1016/j.ajem. 2015.12.012.

16. De'Ath HD, Rourke C, Davenport R, et al. Clinical and biomarker profile of trauma-induced secondary cardiac injury. Br J Surg. 2012;99(6):789-97. https://doi.org/10.1002/bjs.8728.

17. Keskpaik T, Starkopf J, Kirsimagi U, et al. The role of elevated high-sensitivity cardiac troponin on outcomes following severe blunt chest trauma. Injury. 2020;51(5):1177-82. https://doi.org/ 10.1016/j.injury.2019.12.037.

18. Crewdson K, Thompson J, Thomas M. Cardiac troponin T is associated with mortality in patients admitted to critical care in a UK major trauma centre: a retrospective database analysis. J Intensive Care Soc. 2019;20(2):132-7. https://doi.org/10.1177/1751143718 767782.

19. Kalbitz M, Pressmar J, Stecher J, et al. The role of troponin in blunt cardiac injury after multiple trauma in humans. World J Surg. 2017;41(1):162-9. https://doi.org/10.1007/s00268-016-3650-7.

20. Edouard AR, Felten ML, Hebert JL, Cosson C, Martin L, Benhamou D. Incidence and significance of cardiac troponin I release in severe trauma patients. Anesthesiology. 2004;101(6):1262-8. https://doi.org/10.1097/00000542-200412000-00004.

21. Mahmood I, El-Menyar A, Dabdoob W, et al. Troponin T in patients with traumatic chest injuries with and without cardiac involvement: insights from an observational study. N Am J Med Sci. 2016;8(1):17-24. https://doi.org/10.4103/1947-2714.175188. 